\title{
Relato de caso - eventos neuromusculares relacionados com inibidores de checkpoint
}

\author{
Case report - neuromuscular events related to checkpoint \\ inhibitors
}

\section{Informe del caso - eventos neuromusculares relacionados con los inhibidores del punto de control}

\author{
Mariana Queiroz Borges ${ }^{1}$, Ellen Camila Rodrigues Dias ${ }^{2}$, \\ Zeno Augusto de Sousa Neto ${ }^{3}$, Mariana Resende Guedes ${ }^{4}$, \\ Ábner Pereira Prado ${ }^{5}$, Marcos Alexandre Diniz Carneiro ${ }^{6}$
}

\begin{abstract}
1.Estudante de graduação de Medicina do Centro Universitário Alfredo Nasser (UNIFAN), Aparecida de Goiânia-GO, Brasil. ORCID: https://orcid.org/0000-0002-0025-6132

2. Estudante de graduação de Medicina do Centro Universitário Alfredo Nasser (UNIFAN), Aparecida de Goiânia-GO, Brasil. ORCID: https://orcid.org/0000-0003-0682-3668

3.Estudante de graduação de Medicina do Centro Universitário Alfredo Nasser (UNIFAN), Aparecida de Goiânia-GO, Brasil. ORCID: https://orcid.org/0000-0002-2795-4133

4. Estudante de graduação de Medicina do Centro Universitário Alfredo Nasser (UNIFAN), Aparecida de Goiânia-GO, Brasil. ORCID: https://orcid.org/0000-0002-6505-6561

5.Médico Clínico do Instituto de Neurologia de Goiânia. Aparecida de Goiânia-GO, Brasil. ORCID: https://orcid.org/0000-0002-8219-6638

6. Médico Neurologista do Instituto de Neurologia de Goiânia, Goiás, Brasil e Professor Orientador do curso de Medicina do Centro Universitário Alfredo Nasser (UNIFAN). Aparecida de Goiânia-GO, Brasil. ORCID: https://orcid.org/0000-0003-3332-8865
\end{abstract}

\section{Resumo}

O melanoma invasivo possui alta mortalidade e sua incidência tem aumentado nos últimos anos de forma considerável. A descoberta dos inibidores de checkpoint (ICP), melhorou significativamente o prognóstico do tratamento, aumentando sobrevida e qualidade de vida dos pacientes com essas neoplasias. Embora a eficácia comprovada desses tratamentos tenha mudado a história natural do melanoma, vários efeitos colaterais foram relatados, principalmente relacionados ao sistema imunológico. O objetivo desse estudo é descrever um caso de eventos neuromusculares sobrepostos de miopatia, miastenia gravis e neuropatia periférica após início de tratamento com anti-PD1 (Nivolumab) em um paciente com melanoma invasivo.

Unitermos. Miastenia gravis; miopatia; neuropatia; imunoterapia; anti-pd1; melanoma; neoplasia; câncer

\begin{abstract}
Invasive melanoma has high mortality and its incidence has increased considerably in recent years. The discovery of checkpoint inhibitors (PCI) significantly improved treatment prognosis, increasing survival and quality of life patients with these neoplasms. Although the proven efficacy of these treatments has changed the natural history of melanoma, several side effects have been reported, mainly related to the immune system. The aim of this study is to describe a case of overlapping neuromuscular events of myopathy, myasthemia gravis and peripheral neuropathy, anti-PD1 (Nivolumab) treatment in a patient with invasive melanoma.
\end{abstract}

Keywords. Miastenia gravis; myopathy; neuropathy; immunotherapy; anti-pd1; melanoma; neoplasia; cancer 


\section{Resumen}

El melanoma invasivo tiene una elevada mortalidad y su incidencia ha aumentado considerablemente en los últimos años. El descubrimiento de los inhibidores de puntos de control (ICP) ha mejorado significativamente el pronóstico del tratamiento, aumentando la supervivencia y la calidad de vida de los pacientes con estas neoplasias. Aunque la eficacia demostrada de estos tratamientos ha cambiado la historia natural del melanoma, se han notificado varios efectos secundarios, principalmente relacionados con el sistema inmunitario. El objetivo de este estudio es describir un caso de superposición de eventos neuromusculares de miopatía, miastenia gravis y neuropatía periférica tras el inicio del tratamiento anti-PD1 (Nivolumab) en un paciente con melanoma invasivo.

Palabras clave: Miastenia gravis; miopatia; neuropatía; inmunoterapia; anti-pd1; melanoma; neoplasma; cáncer

Trabalho realizado no Centro Universitário Alfredo Nasser (UNIFAN), Aparecida de Goiânia-GO, Brasil.

Conflito de interesse: não

Recebido em: 03/10/2021

Aceito em: 06/12/2021

Endereço para correspondência: Marcos Alexandre Diniz Carneiro. R. T-29 Qd 41, Edifício Excellence, 484. St. Bueno. Goiânia-GO. CEP 74210-050. Fone: (62) 99242-1921. E-mail: marcosdcarneiro@hotmail.com

\section{INTRODUÇÃO}

O melanoma invasivo possui alta mortalidade e sua incidência tem aumentado nos últimos anos de forma considerável. A descoberta dos inibidores de checkpoint (ICP), aprovados desde 2011, melhorou significativamente o prognóstico do tratamento, aumentando sobrevida e qualidade de vida dos pacientes com essas neoplasias. Eles bloqueiam mecanismos de fuga da resposta imune antitumoral, diminuindo a tolerância imunológica contra células neoplásicas. Estão indicados para terapia adjuvante, neoadjuvante e doença metastática. Existem três alvos dos ICP atualmente e incluem o receptor de morte programada 1 (PD-1), o ligante de morte programada 1 (PDL-1) e 0 antígeno 4 associado a linfócito T citotóxico (CTLA4), que podem ser usados em monoterapia ou em combinação entre eles ${ }^{1-3}$.

Embora a eficácia comprovada desses tratamentos tenha mudado a história natural do melanoma e outras 
neoplasias, vários efeitos colaterais foram relatados, principalmente relacionados ao sistema imunológico. Ao atuarem na tolerância imunológica podem induzir eventos imunorrelacionados (IRAEs) em diversos sistemas corporais, principalmente eventos dermatológicos, endócrinos, gastrointestinais e hepáticos. Os eventos graves (grau 3 e 4) são maiores na associação de Ipilimumab com Nivolumab (55\%) em comparação com as drogas isoladamente $(<24 \%)$ e complicações fatais raramente ocorrem $(<1,3 \%)$. Eventos neurológicos também podem acontecer em menor escala, acometendo tanto o sistema nervos central quanto o periférico, com descrições variadas entre 0,8-7,7\% dos pacientes ${ }^{3,4}$.

O objetivo desse estudo é descrever um caso de eventos neuromusculares sobrepostos de miopatia, miastenia gravis e neuropatia periférica após início de tratamento com anti-PD1 (Nivolumab) em um paciente com melanoma invasivo.

\section{RELATO DO CASO}

O presente estudo foi aprovado ao comitê de ética sob o número CAAE: 36012320.0.0000.8011. O consentimento do paciente foi obtido para este manuscrito.

Paciente, sexo masculino, 67 anos, aposentado, procurou atendimento médico com quadro de fraqueza muscular difusa associado a diplopia e ptose palpebral de caráter flutuante. Havia realizado ressecção total de melanoma maligno há 4 meses com margem livre, sem 
metástase linfonodal ou para outros sítios. Como terapia adjuvante estava em uso de Anti - PD1 (Nivolumab) há 2 meses, tendo realizado duas aplicações no total, o que coincidiu com o início dos sintomas atuais.

$\mathrm{Na}$ avaliação neurológica, apresentava força grau 4 difusa sem alterações de reflexos profundos ou patológicos, ptose palpebral a direita e motilidade ocular esquerda comprometida em todas as direções. Exames complementares evidenciavam aumento de enzimas, DHL 423 U/L (125 - 220 U/L), TGP 171 U/L ( 55 U/L) e TGO 97 U/L (05-34 U/L), mas não foi dosado CPK, as enzimas cardíacas e nem colhido líquor. A eletroneuromiografia prévia com estimulação repetitiva compatível com miopatia, sem alterações da junção neuromuscular e tomografia de tórax dentro dos padrões de normalidade. Em outro serviço foi prescrito Piridostigmina $240 \mathrm{mg} /$ dia e Prednisona $20 \mathrm{mg} /$ dia, com alguma resposta sintomática. Devido ao quadro atual, foi submetido a pulsoterapia com metilprednisolona $1 \mathrm{~g} /$ dia por 5 dias e teve melhora parcial.

No retorno, apresentava sintomas residuais já descritos e fraqueza distal do pé direito ("pé caído") como nova manifestação neurológica. Teste do Gelo no olho esquerdo positivo com melhora da ptose. Ressonância de coluna lombar sem alterações importantes, nova eletroneuromiografia sem alterações nervosas, mas com padrão decremental na estimulação repetitiva, anticorpo anti-receptor de acetilcolina aumentado, AChR 12,70 mol/L ( $A$ ChR positivo se $>0,40 \mathrm{~mol} / \mathrm{L}$ ) e USG de nervos ulnares e 
fibulares com espessamento neural difuso e sinais de distrofia dos compartimentos musculares anterior e lateral de ambas as pernas (Figuras 1, 2 e 3 ).

Figura 1. USG Nervo Fibular Comum (NFC) Direito espessado.

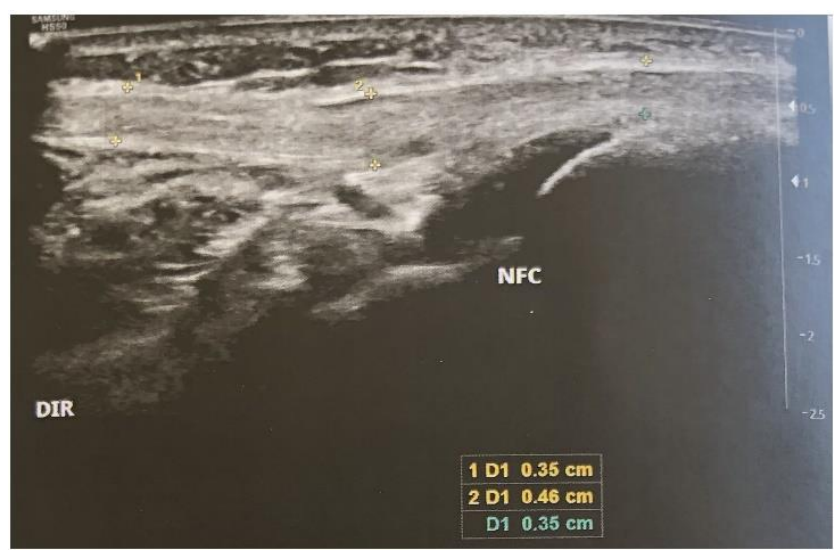

Figura 2. USG NFC Direito e Esquerdo espessados.
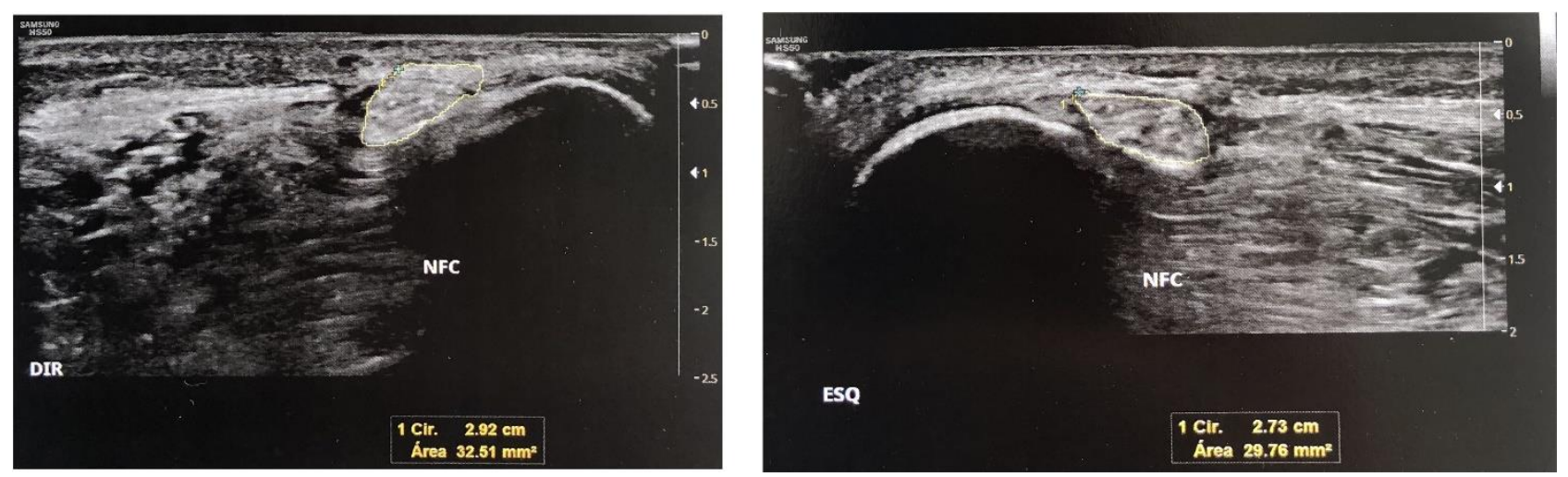

Figura 3. USG Nervo Ulnar Esquerdo e Direito espessados
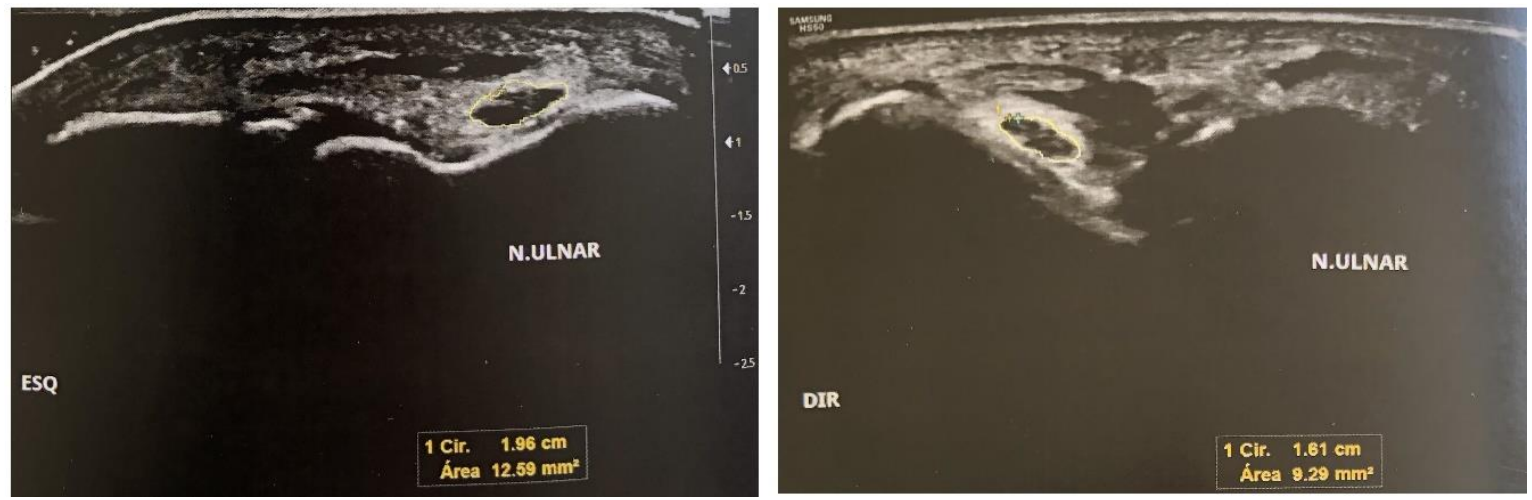
Foi submetido então a infusão de imunoglobulina humana $5 \mathrm{~g} /$ dia por 5 dias e tivemos resposta completa dos sintomas, permitindo desmame progressivo de piridostigmina e prednisona. O paciente encontra-se sem sequelas ou incapacidades. Novos exames dentro dos valores de normalidade e anticorpo ligador do receptor de acetilcolina com diminuição expressiva $(A C h R=3,42 \mathrm{nmol} / \mathrm{L})$.

\section{DISCUSSÃO}

Eventos neurológicos após uso de inibidores de checkpoints são raros, há uma grande parcela de erros diagnósticos e os fatores de risco ainda não são bem conhecidos. Sabe-se que pacientes portadores de doenças autoimunes prévias possuem maior risco de IRAEs após o tratamento com ICP. As alterações neuromusculares são as mais comuns dentre os pacientes neurológicos, representando $60-75 \%$ do total e são representadas principalmente por quadros miopáticos, de miastenia gravis e de neuropatias periféricas, que podem ocorrem isoladamente ou em sobreposição. Embora seja baixa a frequência, a gravidade desses eventos é maior que nos casos não relacionados com ICP levando a sequelas graves ou mesmo óbito dentro dessa população quando não tratados rapidamente $\mathrm{e}^{3-7}$.

Os quadros miopáticos acontecem em pacientes mais idosos, geralmente homens com mais de 70 anos e têm início nos 2 primeiros meses após a primeira aplicação de 
ICP, sendo esse tempo menor em pacientes com terapia combinada. A apresentação clínica varia de aumentos isolados de CPK até fraqueza generalizada e acomete musculatura ocular, facial, bulbar, axial e de membros. A doença tem evolução em menos de um mês, podendo levar a insuficiência respiratória e miocardiopatia, duas complicações que podem ser fatais. As enzimas musculares, que incluem $\mathrm{CPK}$, troponina e transaminases, estão aumentadas e permanecem assim até 3 meses do início do diagnóstico. Anticorpos específicos são negativos na grande maioria dos pacientes. A eletroneuromiografia geralmente está alterada, revelando padrão miopático e a biópsia muscular demonstra infiltrado inflamatório com graus variados de miopatia necrotizante, o que pode ser alterado após instalação de corticoterapia ${ }^{7-11}$.

A miastenia gravis, uma complicação mais rara que os quadros miopáticos isolados, pode corresponder a exarcebações de doenças prévias ou diagnósticos novos. Estudos de séries de casos indicam que $<1 \%$ apresentou esse efeito colateral, porém a maioria com sintomas graves e classificação maior que 3 da Myasthenia Gravis Foundation of America (MGFA), sendo que $>40 \%$ necessitam de ventilação mecânica. Os sintomas ocorrem nas primeiras doses da medicação, especialmente depois das duas primeiras aplicações, mas há relatos após 15 semanas do início do tratamento. Os anticorpos Anti-AChR podem estar aumentados antes mesmo dos sintomas de fraqueza muscular, são importantes para o diagnóstico e se 
correlacionam com o tratamento. Porém, há casos seronegativos que podem ser diagnosticados pela eletroneuromiografia e outros testes funcionais (teste do gelo ou da piridostigmina). Anticorpos Anti-MUSK geralmente são negativos ${ }^{11-14}$.

Devemos destacar as complicações cardíacas que são a principal causa de mortalidade entre os pacientes com IRAEs. Miocardite ocorre entre 0,27-1,14\%, mas a frequência é bem maior nos casos de alterações neuromusculares ( $25 \%$ nos casos de miopatia e $11 \%$ nos casos de miastenia gravis) e todos os pacientes deveriam ser rastreados. Geralmente, os sintomas aparecem dentro de 30 dias do início da terapia com ICP e são diagnosticados pelo quadro clínico associado a alterações de enzimas cardíacas, eletrocardiográficas, ecocardiográficas ou de ressonância magnética. O principal fator de risco assim como fator de mal prognóstico é a associação de inibidores de checkpoint. A mortalidade chega a $50 \%$ dos $\operatorname{casos}^{15}$.

Neuropatias periféricas são pouco descritas na literatura, representadas principalmente por relatos de casos isolados. Uma série com maior número de pacientes que foram encaminhados para a realização de eletroneuromiografia devido sintomas há menos de 100 dias do início de terapia com ICP foi descrita recentemente. Dentre os 23 pacientes encaminhados, encontrou-se os seguintes tipos de neuropatias: neuropatia desmielinizante, polineuropatia sensoriomotor axonal, neuropatia axonal 
sensitiva e mononeuropatia múltipla. Alguns pacientes tiveram alterações sugestivas de Polineuropatia desmielinizante inflamatória crônica (PDIC). Alterações liquóricas ocorreram em 5 pacientes, tanto aumento de proteínas quanto aumento celular. Nenhum desses pacientes tinham doença prévia devido uso de quimioterápicos, porém alguns estavam em uso concomitante com imunoterapia. A mediana de início dos sintomas foi de 20 dias, com variação entra 2 a 94 dias. A maioria melhorou com o tratamento imunoterápico instituído ${ }^{16}$.

Segundo os guidelines atuais, o tratamento dos IRAEs envolve a interrupção dos ICP associado a imunoterapia, sendo a corticoterapia a primeira escolha. Nos casos refratários, a imunoglobulina humana ou plasmaferese estão indicadas. Ainda faltam estudos clínicos a respeito do assunto, sendo as recomendações baseadas em series de casos e opiniões de especialistas. A grande maioria dos pacientes respondem a imunoterapia, com melhora progressiva que podem durar dias até meses nos casos mais graves e não precisam de imunossupressão contínua. Após melhora, pacientes selecionados podem ser retratados com ICP a depender da necessidade terapêutica da neoplasia de base, avaliando risco e benefícios da decisão ${ }^{17-}$ 19 


\section{CONCLUSÕES}

Relatamos um caso de eventos neuromusculares sobrepostos após terapia com Nivolumab para tratamento adjuvante de melanoma. Embora grande parte dos pacientes tenham sintomas graves, nosso paciente teve fraqueza generalizada, mas sem necessidade de internação ou suporte ventilatório. A rápida instalação de corticoterapia e imunoglobulina devido sintomas refratários podem justificar a boa evolução. O caso é interessante por demonstrar a possibilidade de sobreposição de eventos neuromusculares como IRAEs, sendo necessário investigações amplas nesses tipos de pacientes. A instalação de protocolos clínicos é importante, já que há vários diagnósticos diferenciais confundidores nesses casos.

\section{REFERÊNCIAS}

1.Schadendorf D, van Akkooi ACJ, Berking C, Griewank KG, Gutzmer $\mathrm{R}$, Hauschild A, et al. Melanoma. Lancet 2018;392:971-84. https://doi.org/10.1016/S0140-6736(18)31559-9

2. Herrscher $\mathrm{H}$, Robert $\mathrm{C}$. Immune checkpoint inhibitors in melanoma in the metastatic, neoadjuvant, and adjuvant setting. Curr Opin Oncol 2020;32:106-13. https://doi.org/10.1097/cco.0000000000000610

3. Hottinger AF. Neurologic complications of immune checkpoint inhibitors. Curr Opin Neurol 2016;29:806-12.

https://doi.org/10.1097/wco.0000000000000391

4.Wang DY, Salem JE, Cohen JV, Chandra S, Menzer C, Ye F, et al. Fatal Toxic Effects Associated With Immune Checkpoint Inhibitors: A Systematic Review and Meta-analysis. JAMA Oncol 2018;4:1721-8. https://doi.org/10.1001/jamaoncol.2018.3923

5. Kao JC, Brickshawana A, Liewluck T. Neuromuscular Complications of Programmed Cell Death-1 (PD-1) Inhibitors. Curr Neurol Neurosci Rep 2018;18:63. https://doi.org/10.1007/s11910-018-0878-7

6.Johansen A, Christensen SJ, Scheie D, Højgaard JLS, Kondziella D. Neuromuscular adverse events associated with anti-PD-1 monoclonal antibodies: Systematic review. Neurology 2019;92:663-74. https://doi.org/10.1212/wnl.0000000000007235 
7.Johnson DB, Sullivan RJ, Ott PA, Carlino MS, Khushalani NI, Ye F, et al. Ipilimumab Therapy in Patients With Advanced Melanoma and Preexisting Autoimmune Disorders. JAMA Oncol 2016;2:234-40. https://doi.org/10.1001/jamaoncol.2015.4368

8. Touat M, Maisonobe T, Knauss S, Hadj B, Salem O, Hervier B, et al. Immune checkpoint inhibitor-related myositis and myocarditis in patients with cancer. Neurology 2018;91:e985-94. https://doi.org/10.1212/wnl.0000000000006124

9.Anquetil C, Salem JE, Lebrun-Vignes B, Johnson DB, Mammen AL, Stenzel W, et al. Immune Checkpoint Inhibitor-Associated Myositis: Expanding the Spectrum of Cardiac Complications of the Immunotherapy Revolution. Circulation 2018;138:743-5. https://doi.org/10.1161/circulationaha.118.035898

10.Shah M, Tayar JH, Abdel-Wahab N, Suarez-Almazor ME. Myositis as an adverse event of immune checkpoint blockade for cancer therapy. Semin Arthritis Rheum 2019;48:736-40. https://doi.org/10.1016/j.semarthrit.2018.05.006

11.Seki M, Uruha A, Ohnuki Y, Kamada S, Noda T, Onda A, et al. Inflammatory myopathy associated with PD-1 inhibitors. J Autoimmun 2019;100:105-13.

https://doi.org/10.1016/j.jaut.2019.03.005

12. Moreira A, Loquai C, Pföhler C, Kähler KC, Knauss S, Heppt MV, et al. Myositis and neuromuscular side-effects induced by immune checkpoint inhibitors. Eur J Cancer 2019;106:12-23. https://doi.org/10.1016/j.ejca.2018.09.033

13. Makarious D, Horwood K, Coward JIG. Myasthenia gravis: An emerging toxicity of immune checkpoint inhibitors. Eur J Cancer 2017;82:128-36. https://doi.org/10.1016/j.ejca.2017.05.041

14.Suzuki S, Ishikawa N, Konoeda F, Seki N, Fukushima S, Takahashi $\mathrm{K}$, et al. Nivolumab-related myasthenia gravis with myositis and myocarditis in Japan. Neurology 2017;89:1127-34. https://doi.org/10.1212/wnl.0000000000004359

15. Hu JR, Florido R, Lipson EJ, Naidoo J, Ardehali R, Tocchetti CG, et al. Cardiovascular toxicities associated with immune checkpoint inhibitors. Cardiovasc Res 2019;115:854-68. https://doi.org/10.1093/cvr/cvz026

16.Chen X, Haggiagi A, Tzatha E, DeAngelis LM, Santomasso B. Electrophysiological findings in immune checkpoint inhibitor-related peripheral neuropathy. Clin Neurophysiol 2019;130:1440-5. https://doi.org/10.1016/j. clinph.2019.03.035

17.Puzanov I, Diab A, Abdallah K, Bingham CO 3rd, Brogdon C, Dadu $\mathrm{R}$, et al. Managing toxicities associated with immune checkpoint inhibitors: consensus recommendations from the Society for Immunotherapy of Cancer (SITC) Toxicity Management Working Group. J Immunother Cancer 2017;5:95. https://doi.org/10.1186/s40425-017-0300-z

18.Brahmer JR, Lacchetti C, Schneider BJ, Atkins MB, Brassil KJ, Caterino JM, et al. Management of Immune-Related Adverse Events in Patients Treated With Immune Checkpoint Inhibitor Therapy: 
American Society of Clinical Oncology Clinical Practice Guideline. J Clin Oncol 2018;36:1714-68.

https://doi.org/10.1200/jco.2017.77.6385

19.Thompson JA, Schneider BJ, Brahmer J, Andrews S, Armand P, Bhatia S, et al. Management of Immunotherapy-Related Toxicities, Version 1.2019. J Natl Compr Canc Netw 2019;17:255-89. https://doi.org/10.6004/jnccn.2019.0013 